\title{
Effect of Scale on Freely Propagating Flames in Aluminum Dust Clouds
}

\author{
Philippe Julien ${ }^{a, b}$, James Vickery ${ }^{a}$, Sam Whiteley $^{a}$, Alex Wright ${ }^{a}$, Sam Goroshin ${ }^{a}$, Jeffrey M. \\ Bergthorson $^{a}$, \& David L. Frost ${ }^{a}$ \\ E-mail:philippe.julien@mail.mcgill.ca \\ ${ }^{a}$ McGill University, 817 Sherbrooke St West, Montreal, Quebec, Canada, H3A 0C3 \\ ${ }^{\mathrm{b}}$ Corresponding author: philippe.julien@mail.mcgill.ca, 1-514-991-4191
}

\begin{abstract}
The majority of experimental tests done on combustible dusts are performed in constant volume vessels that have limited or no optical access. Over the years, McGill University has been developing alternative experimental techniques based on direct observation of dust flames, yielding reliable fundamental parameters such as flame burning velocity, temperature and structure. The present work describes two new experimental set-ups allowing direct observation of isobaric and freely propagating dust flames at two sufficiently different scales to test the influence of scale on dust flame phenomena. In the laboratory-scale experiments, a few grams of aluminum powder are dispersed in transparent, $30 \mathrm{~cm}$ diameter latex balloons that allow for full visualization of the spherical flame propagation. In the field experiments, about $1 \mathrm{~kg}$ of aluminum powder is dispersed by a short pulse of air, forming a conical dust cloud with a total volume of about $5 \mathrm{~m}^{3}$. High-speed digital imaging is used to record the particle dispersal and flame propagation in both configurations. In the small-scale laboratory tests, the measured flame speed is found to be about $2.0 \pm 0.2 \mathrm{~m} / \mathrm{s}$ in fuel-rich aluminum clouds. The burning velocity, calculated by dividing the measured flame speed by the expansion factor deduced from thermodynamic equilibrium calculations, correlates well with the previously measured burning velocity of about $22-24 \mathrm{~cm} / \mathrm{s}$ from Bunsen dust flames. Flame speeds observed in field experiments with large-scale clouds, however, are found to be much higher, in the range of $12 \pm 2$ $\mathrm{m} / \mathrm{s}$. Estimations are presented that show that the presumably greater role of radiative heat transfer in larger-scale aluminum flames is insufficient to explain the six-fold increase in flame speed. The role of residual large-eddy turbulence, as well as the frozen-turbulence effect leading to large-scale dust concentration fluctuations that cause flame folding, are discussed as two possible sources for the greater flame speed.
\end{abstract}

Keywords: aluminum dust cloud, flame speed, scale, radiation heat transfer, turbulence 


\section{Introduction}

The ever increasing role of modern technologies based on metal powders, such as powder metallurgy, chemical processing, plasma spraying, etc., and the rapidly increasing scale of metal powder production are overshadowed by the increased human and material cost of accidents caused by metal dust explosions (Cashdollar\& Hertzberg, 1987). In spite of the strong impetus to implement state-of-the-art preventive measures to mitigate accidents, the progress in this field has been relatively slow in comparison to other branches of preventive science. The slow progress in prevention of metal dust explosions reflects the relatively underdeveloped state of combustion science in this field in contrast to the impressive progress achieved in understanding the physics and chemistry of homogeneous gas flames.

The primary reason for the slow progress in dust combustion science is rooted in the difficulties of extracting the fundamental combustion parameters, such as ignition temperature, burning velocity, flame quenching distance and flame structure, from laboratory experiments with metal dust clouds. Traditionally, most dust combustion tests are performed in constant volume vessels. Though convenient for empirical testing, constant-volume bombs have limited or no optical access, and the pressure rise is the only parameter typically measured in most experiments. The pressure history provides limited insight into the dust flame propagation since the deduction of the flame speed from the rate of pressure rise is neither accurate nor representative if the flame propagation deviates from the ideal picture of a spherically symmetric laminar flame (Hertzberg, Cashdollar, \&Zlochower, 1998; Pu, Jia, Wand, \&Skjold, 2007). Some experiments have demonstrated that the residual turbulence induced in the mixture during the dust dispersal phase may have a considerable influence on flames ignited with short delay times after dispersal (Dahoe, Cant, \& Scarlett, 2001; Kang Pu, Jarosinski, Sheng Tai, Kauffman, \&Sichel, 1989). Furthermore, the implementation of modern flame diagnostic techniques, such as emission and laser-absorption spectroscopy, is required to elucidate the flame structure and verify equilibrium calculations of flame temperatures against experimental values, which is difficult to accomplish in constant volume bombs.

An array of experimental set-ups allowing for visual observation of flames in dust and hybrid combustible gas-dust clouds has been developed at McGill University over the past two decades. They are based on experimental methods that have proven to be successful in gas flame research and include Bunsen dust burners (Goroshin, Fomenko, \& Lee, 1996a; Goroshin, Mamen, Higgins, Bazyn, Glumac, \&Krier, 2007; Julien, Soo, Goroshin, Frost, Bergthorson, Glumac, Zhang, 2014a; Julien, Whiteley, Goroshin, Soo, Frost, Bergthorson, 2014b; Soo, Julien, Goroshin, Bergthorson, \& Frost, 2013), counter-flow dust burners (under development), as well as dust flames propagating in transparent tubes and narrow channels (Palecka, Julien, Goroshin, Bergthorson, Frost, \& Higgins, 2014; Tang, Goroshin, Higgins, \& Lee, 2009). These set ups have permitted an accurate measurement of the burning velocities (Goroshin et al., 1996a), flame quenching distances (Goroshin, Bidabadi, \& Lee, 1996b) and spectroscopic diagnostics of the dust flame structure (Goroshin et al., 2007) in dust and hybrid combustible gas-dust mixtures (Julien et al., 2014a; Julien et al., 2014b). A large series of dust combustion experiments were performed at low gravity on board a parabolic flight aircraft (Goroshin, Tang, Higgins, \& Lee, 2011; Tang, Goroshin, \& Higgins, 2011; Tang et al., 2009). By eliminating particle 
sedimentation and natural convection, microgravity conditions have permitted the observation of laminar dust flames for a very wide range of particle sizes. The current paper presents two new experimental systems developed to investigate the effects of scale on the flame propagation in combustible dust clouds. The first apparatus, which is similar to the one recently reported by Skjold, Olsen, and Castellanos (2013), allows the laboratory observation of isobaric spherical flames in dust clouds dispersed in transparent latex balloons with an initial volume of about 14 litres. The second apparatus permits the creation of unconfined dust clouds with a total volume in the range of $5-10 \mathrm{~m}^{3}$.

\section{Experimental Methods and Results}

\subsection{Small-scale spherical dust flames in transparent latex balloons}

Figure 1 shows a photograph of the experimental apparatus, including the dispersion and ignition systems (the control unit, mixing tank and safety housing are not visible). The balloon is placed on the neck of the balloon holder, which has three different ports: one for inflating the balloon, one for dispersing the powder and one for the igniter. At the start of a test, the balloon is inflated through a solenoid valve controlled by a remote timer. Once the balloon reaches the desired size, about $30 \mathrm{~cm}$ in diameter, a calibration image is taken. The powder, placed in a receptacle within the dispersion unit, is then injected into the balloon. The injection is initiated by the opening of a second solenoid valve, causing a high-pressure gas jet to impinge on the powder surface, lofting the powder, and entraining it into the flow through a perforated cap and into the balloon. This method results in a uniform dispersion of the powder throughout the balloon. The two-phase flow is initially turbulent, with prominent, large-scale vortices moving primary along the wall of the balloon. A delay is applied to allow the turbulence to decay before igniting the mixture by discharging a capacitor through a 100 micron thick tungsten wire positioned at the center of the balloon. A small, yet unavoidable, amount of powder is deposited on the surface of the balloon during the powder dispersal process, making the effective concentration somewhat lower than the one calculated based on the initial powder mass and the volume of the inflated balloon. It is estimated that the amount of deposited powder does not exceed $10 \%$ of the initial powder weight in the receptacle.

The transparent balloon allows for the imaging of all experimental stages with a high-speed camera, including powder dispersal, ignition, and the subsequent isobaric flame propagation. The dust dispersal process, with the balloon uniformly backlit with a diffuse light source, is recorded at 300 frames per second using a Photron SA5 camera. The flame propagation process is recorded with the same camera typically at 5,000 frames per second. 


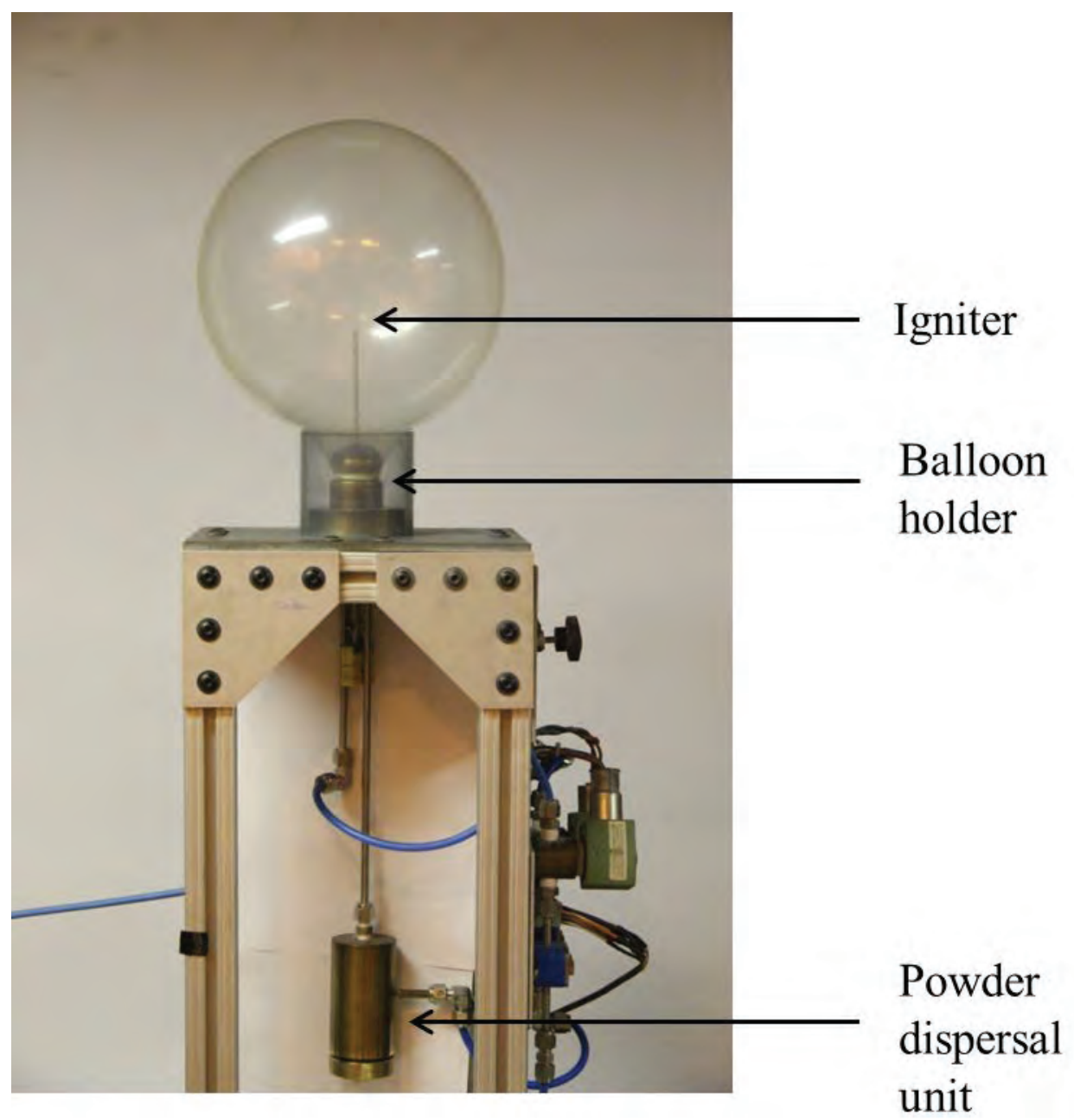

Figure 1: Small-scale apparatus.

This method of measuring flame speed is first validated using methane-air mixtures of various equivalence ratios. The contour of the flame on the high-speed movie is traced for every frame and the radius is taken to be the average distance between the contour and the center-of-mass. The flame speed is equal to the slope of the curve, which is found by linear regression.

Conservation of mass allows the calculation of the 1D laminar burning velocity, $S_{L}(\mathrm{Law}, 2006)$ using $S_{L}=S_{f}\left(\rho_{b} / \rho_{u}\right)$, where $S_{f}$ is the flame speed, and $\rho_{b}$ and $\rho_{u}$ are the burned and unburned gas densities, respectively. The density of the gas before and after combustion is found using the chemical equilibrium solver Cantera (Goodwin, 2003). As shown in Fig. 2, the burning velocities are in good agreement with other literature values measured with spherical flames (Gu, Haq, Lawes, \&Wooller, 2000; Hassan, Aung, \&Faeth, 1998; Qin, \&Ju, 2005). 


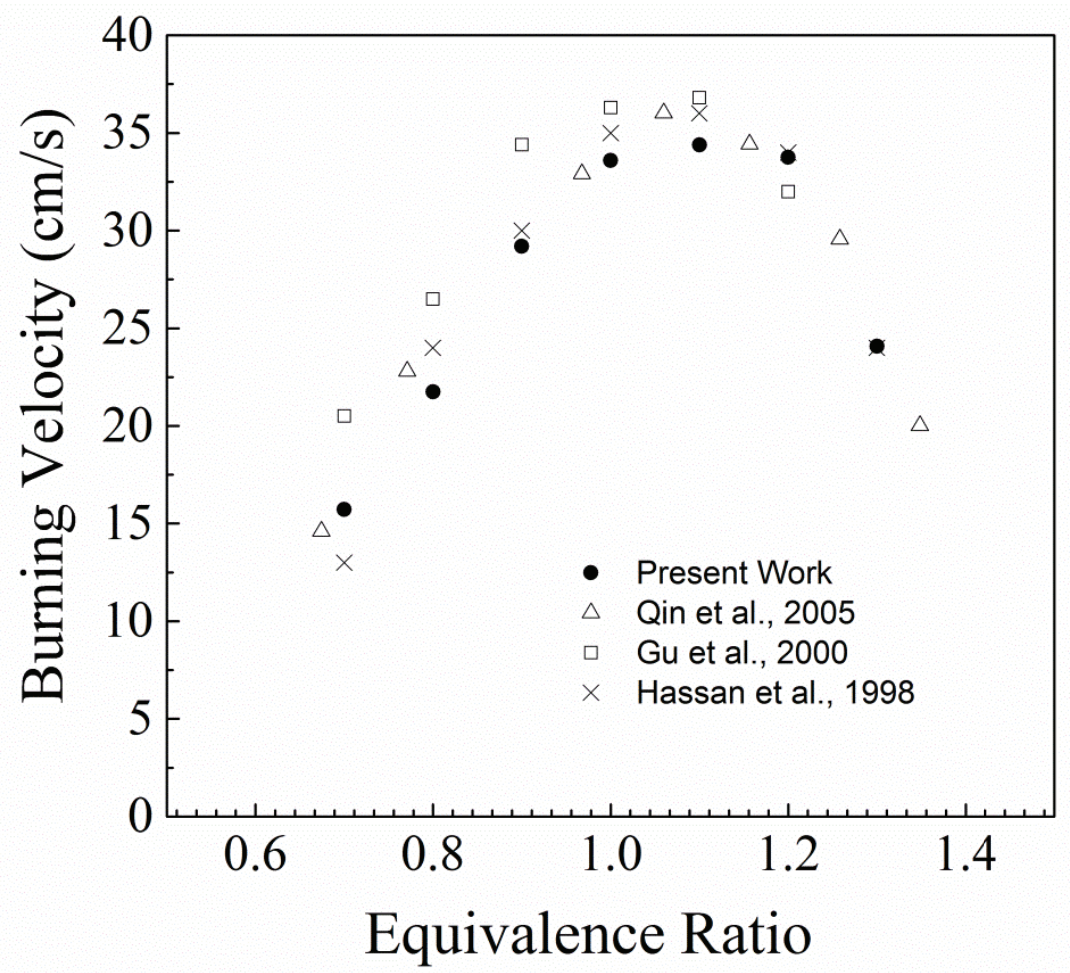

Figure 2: Burning velocity vs. equivalence ratio for methane-air mixtures compared to results from other freely-propagating spherical flames.

Tests with stoichiometric methane-air mixtures are performed to determine the minimum delay time required between the powder dispersal process and ignition. A pulse of pre-mixed methaneair instead of air is introduced into the balloon with no powder to study the effect of turbulence on the flame speed. The results are shown in Fig. 3 and the dashed line represents the laminar flame speed. It can be seen that approximately 5 seconds are required for the effect of turbulence to be negligible. This delay time is found to be a good compromise between the decay of turbulence and minimal settling of the powder. 


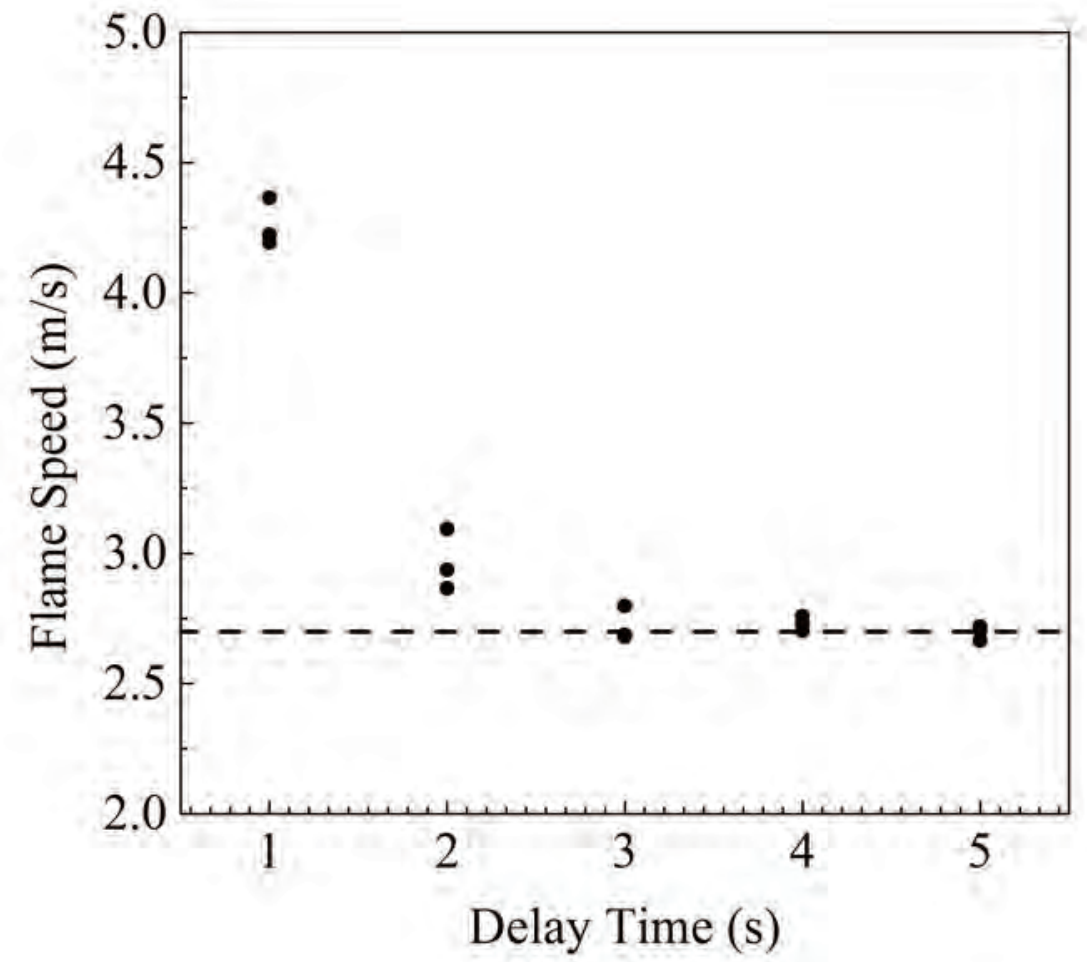

Figure 3: Methane flame speed as a function of ignition delay time. The dashed line represents the laminar flame speed.

Laboratory-scale experiments with aluminum powder are carried out with Ampal aluminum powder (Ampal 637, Ampal, NJ), which has a Sauter mean diameter $d_{32}$ of about 5.6 microns. An electron microscope picture of the powder and the particle size distribution can be found in a recent publication (Julien et al., 2014). The same batch of Ampal powder has been used in previous experiments with stabilized Bunsen aluminum flames (Goroshin, Fomenko, \& Lee, 1996). This allows for direct quantitative comparison with the burning velocities obtained in this work. Figure 4 shows still frames during the dispersal of $0.5 \mathrm{~g}$ of aluminum powder within the latex balloon. The final dust mass concentration is about $75 \mathrm{~g} / \mathrm{m}^{3}$. 

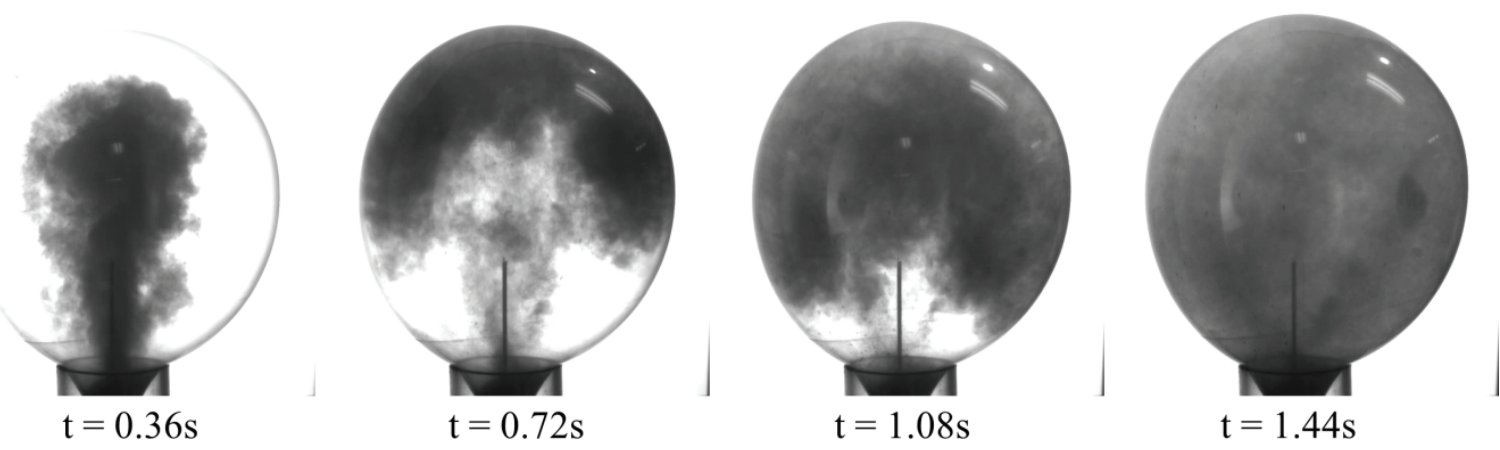

Figure 4: Dispersal of $0.5 \mathrm{~g}$ of aluminum within a transparent balloon. Though fully dispersed at the time of $1.44 \mathrm{~s}$, an additional delay is applied prior to ignition to allow the turbulence to decay.

Figure 5 shows the flame propagation through an aluminum dust cloud with a concentration of about $500 \mathrm{~g} / \mathrm{m}^{3}$. The images are processed the same way as for the methane flames. A typical result for the average radius as a function of time is shown in Fig. 6, with the slope corresponding to the flame speed.

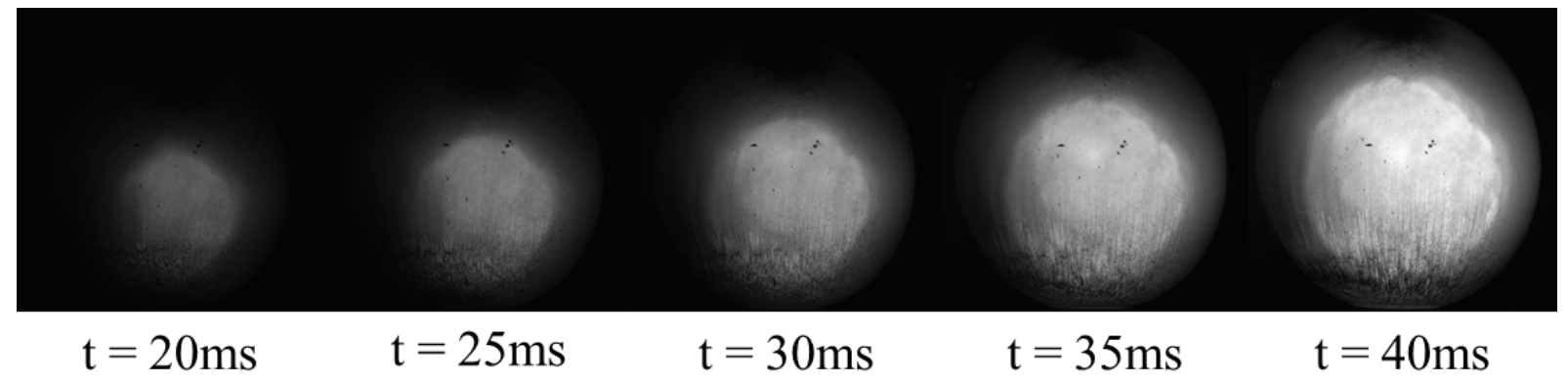

Figure 5: Characteristic aluminum flame propagation for a powder concentration of $500 \mathrm{~g} / \mathrm{m}^{3}$. 


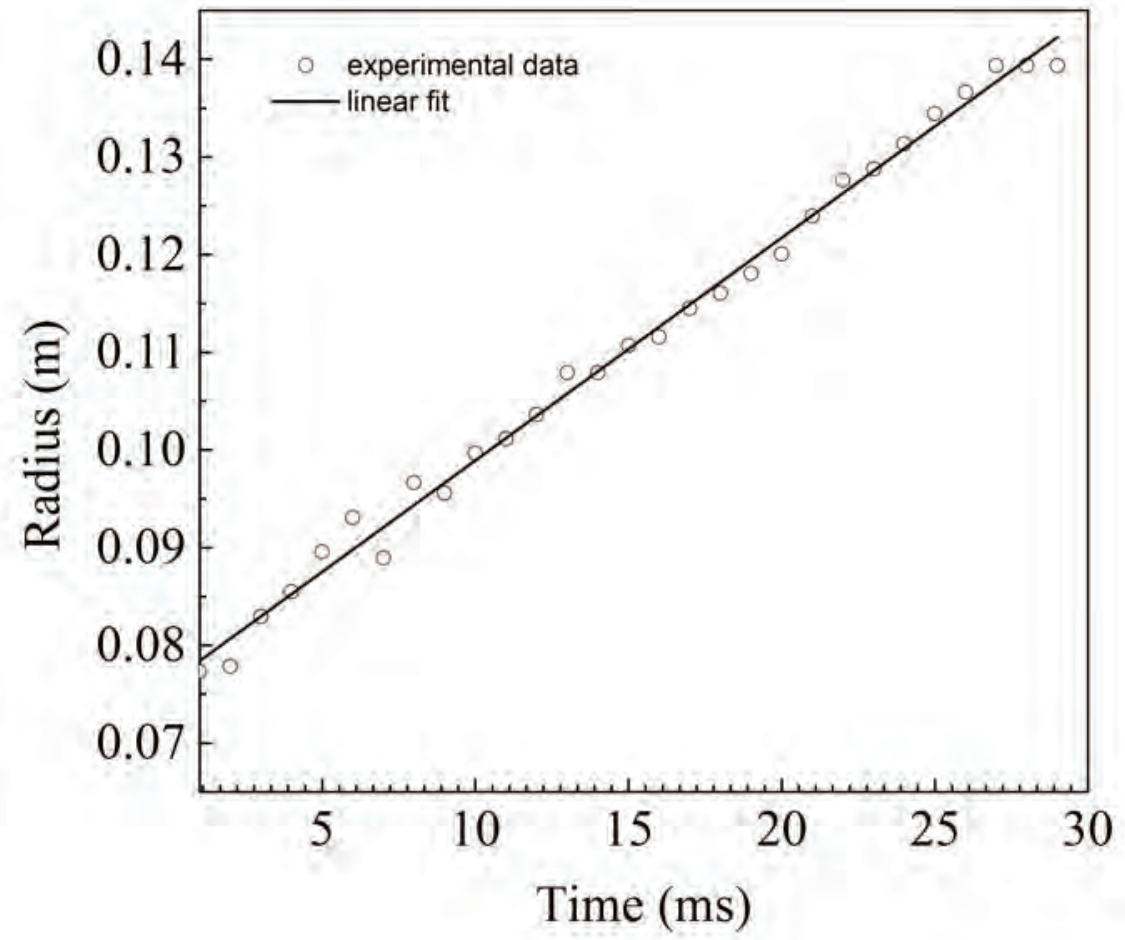

Figure 6: Flame front radius as a function of time for flame propagation in an aluminum-air mixture

The flame speed as a function of aluminium concentration is shown on Fig. 7. The burning velocity is obtained by multiplying the flame speed by the ratio of the burnt and unburnt densities and is shown in Fig. 8 with solid square symbols and compared to the results found by Goroshin et al. (1996a) on the Bunsen burner, corresponding to the open circle symbols. The results are in good agreement with burning velocities on the order of $20 \mathrm{~cm} / \mathrm{s}$. 


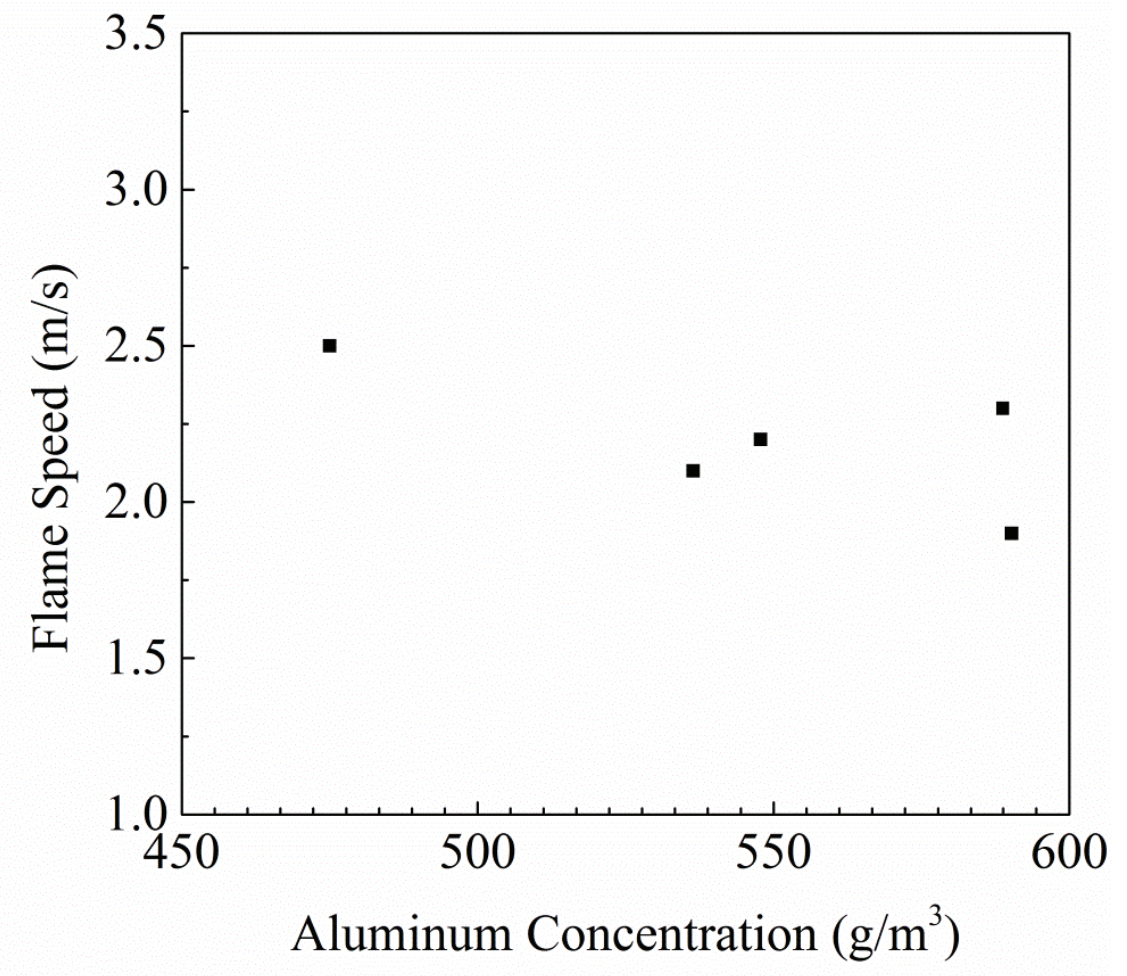

Figure 7: Measured flame speed for various aluminum concentrations

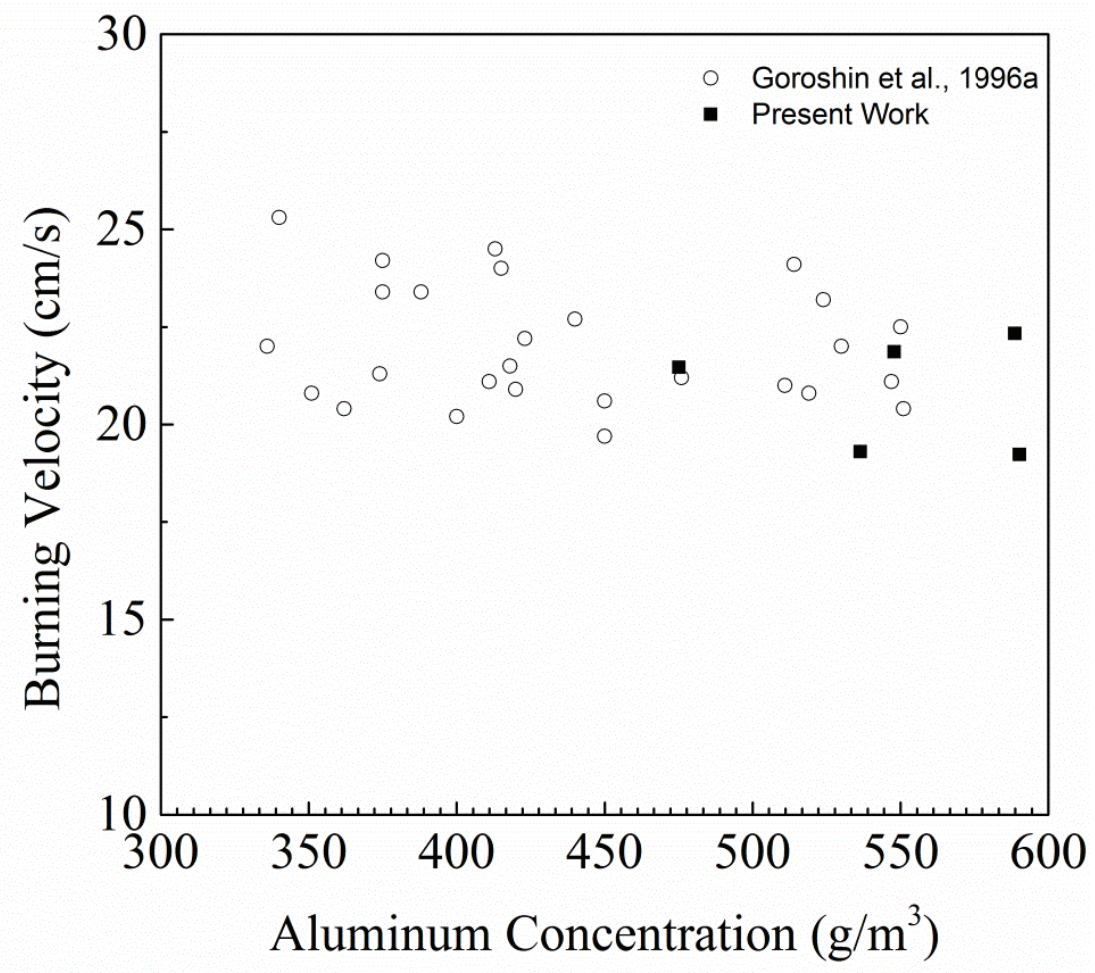


Figure 8: Measured flame speed for various aluminum concentrations from present investigation compared with previous work using the same powder, but with a stabilized Bunsen aluminum dust-air flame

\subsection{Field experiments with large-scale dust clouds}

Figure 9 shows the dust dispersal apparatus specifically designed for the field tests. The apparatus can disperse up to $1 \mathrm{~kg}$ of metal powder within 2-3 seconds, creating a large dust cloud with an average aluminum dust concentration close to the stoichiometric value, i.e., 300 $\mathrm{g} / \mathrm{m}^{3}$. The lightly compacted column of aluminum powder is held in a cylindrical metallic cartridge and is pushed up as a whole towards the dispersal nozzle by a pneumatic piston at a constant rate. The dust is dispersed at the base of the nozzle by a sonic annular air jet, referred to as an air-knife, from a $0.5 \mathrm{~mm}$ annular slot. Figure 10 shows the powder dispersal process. The dispersed powder forms a vertical dust cloud with a conical shape that is about $4 \mathrm{~m}$ tall and up to 2 meters wide at the top, as shown on Fig. 10. The cloud is ignited by a small 2-3 g charge of black powder placed in an open metal tube, immediately after the dispersal air is cut-off by a solenoid valve. The powder dispersal and flame propagation processes were visualized with a Photron SA5 high-speed camera operating at 1,000 frames per second. Fig. 11 shows a series of single frames at different times illustrating the flame propagation through the dust cloud. A single frame from Fig. 11 is shown in Fig. 12 to identify the different regions of the flame. Convection of the dust cloud due to the ambient wind conditions during the field trials, even for low wind speeds, made it impossible to introduce a significant delay time between the dispersal and ignition events. 


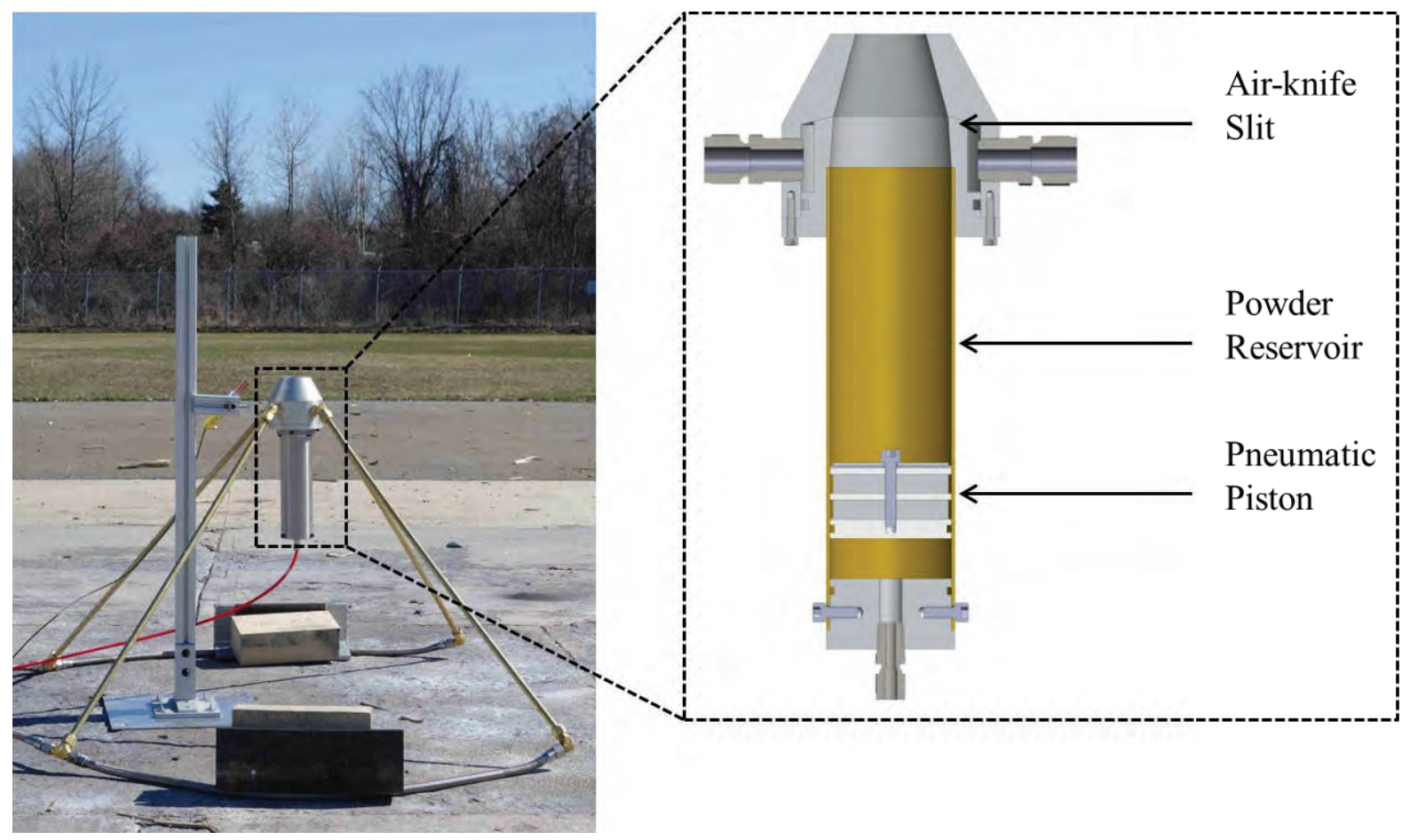

Figure 9: Photograph of field test apparatus and schematic of powder dispersal unit.

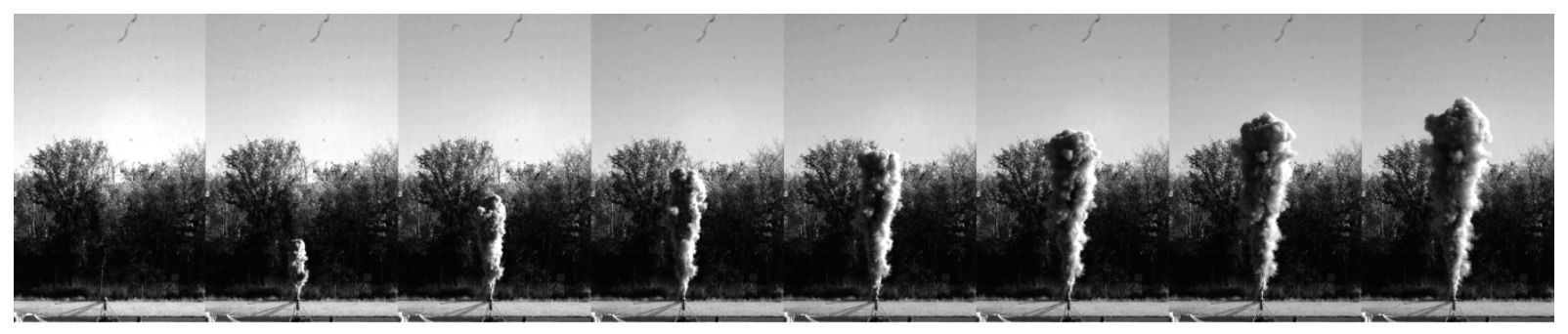

Figure 10: Dispersal of aluminum dust to form a conical-shaped dust cloud

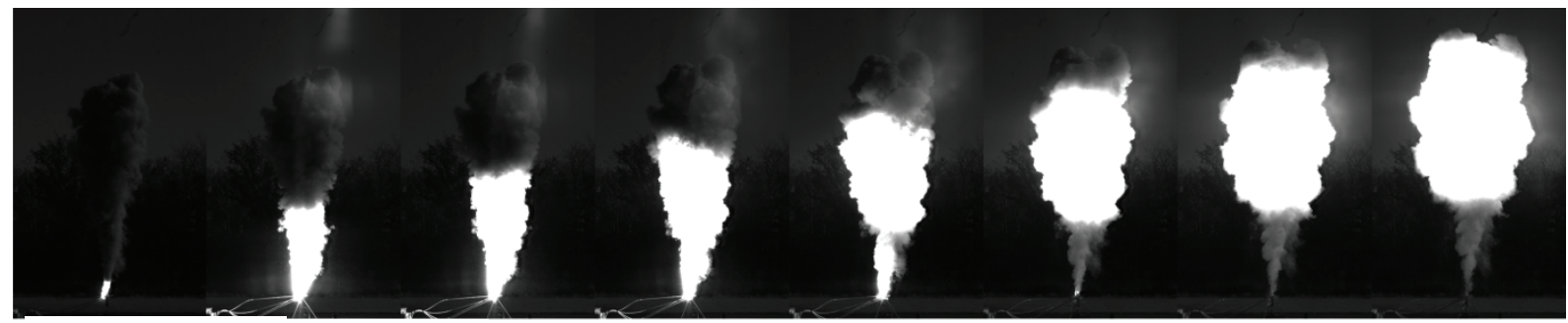

Figure 11: Flame propagation for cloud shown in Fig. 10 


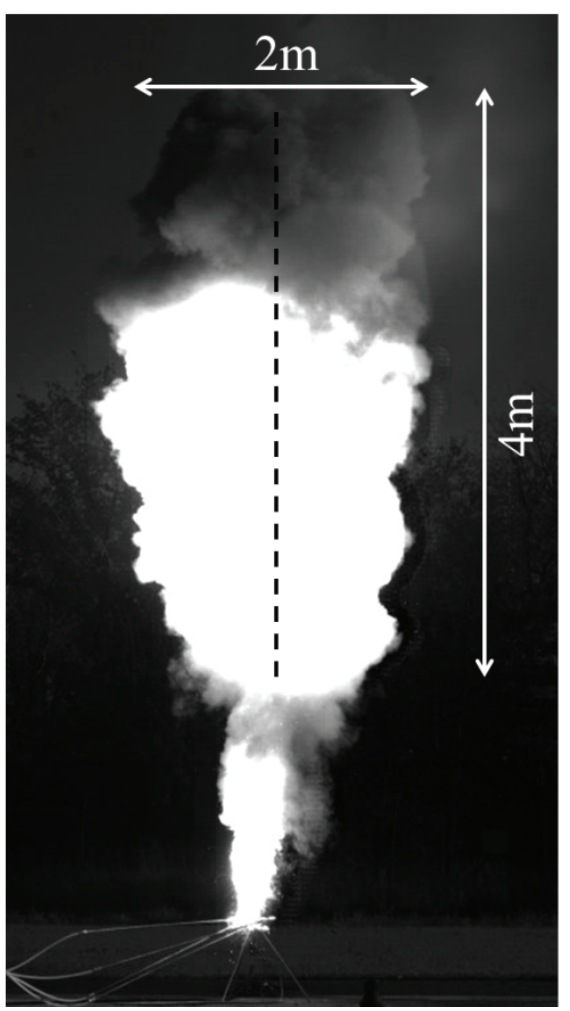

Figure 12: Still frame of the aluminum flame (top) propagating upwards, taken from Fig. 11 at a time of $200 \mathrm{~ms}$ after initiation of flame propagation; narrow flame in the lower part of the flame is the remnant of the black powder charge. The dashed line indicates the center of the cloud.

The progression of the flame front can be determined by tracing the location of the leading edge of the flame. Light scattered from the aluminum particles ahead of the flame makes it difficult to precisely determine the front boundary and this issue is accounted for in the experimental uncertainty. Figure 13 shows the vertical progression of the flame front for the trial shown in Fig. 11. Figure 14 shows the position of the flame front as a function of height for a vertical crosssection. The flame speed is found by a linear fit through these data points. As shown in Fig. 15, the average flame speed at different horizontal positions across the cloud varies by an insignificant amount. 


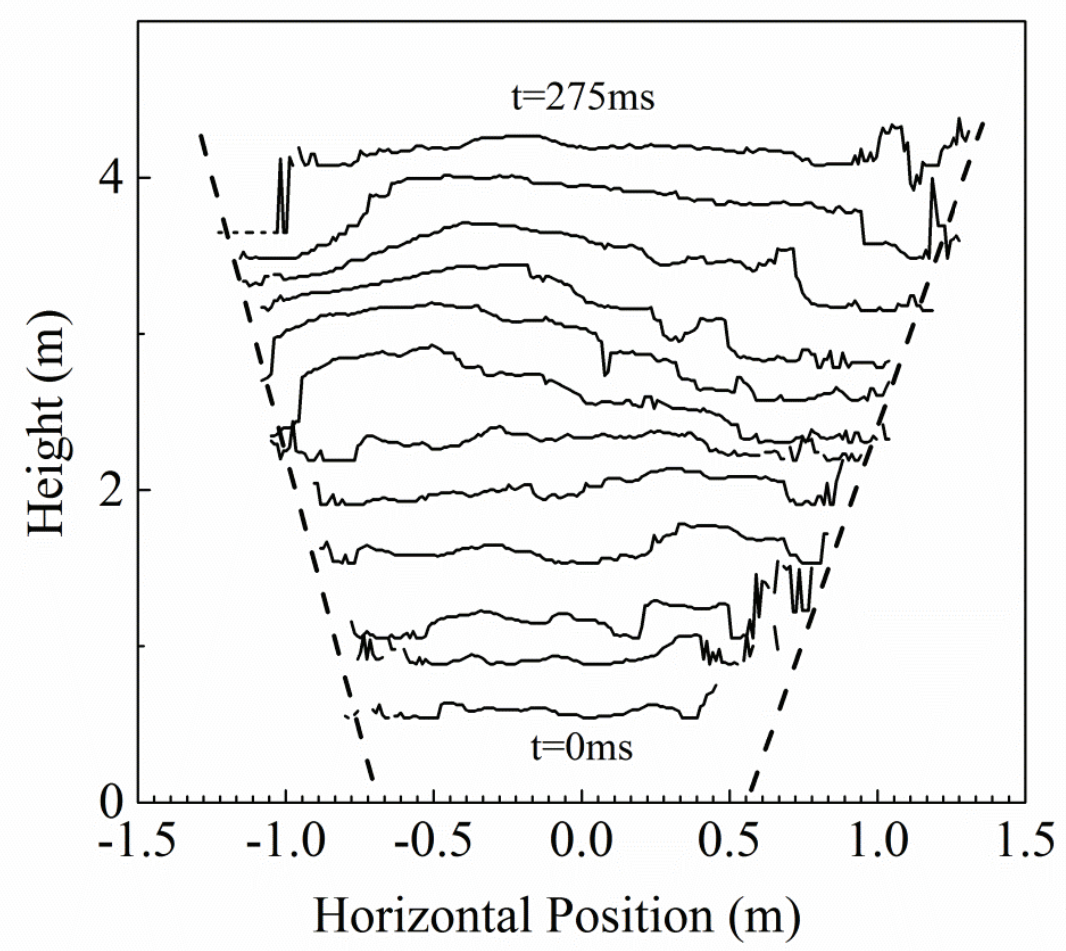

Figure 13: Flame front progression over time.

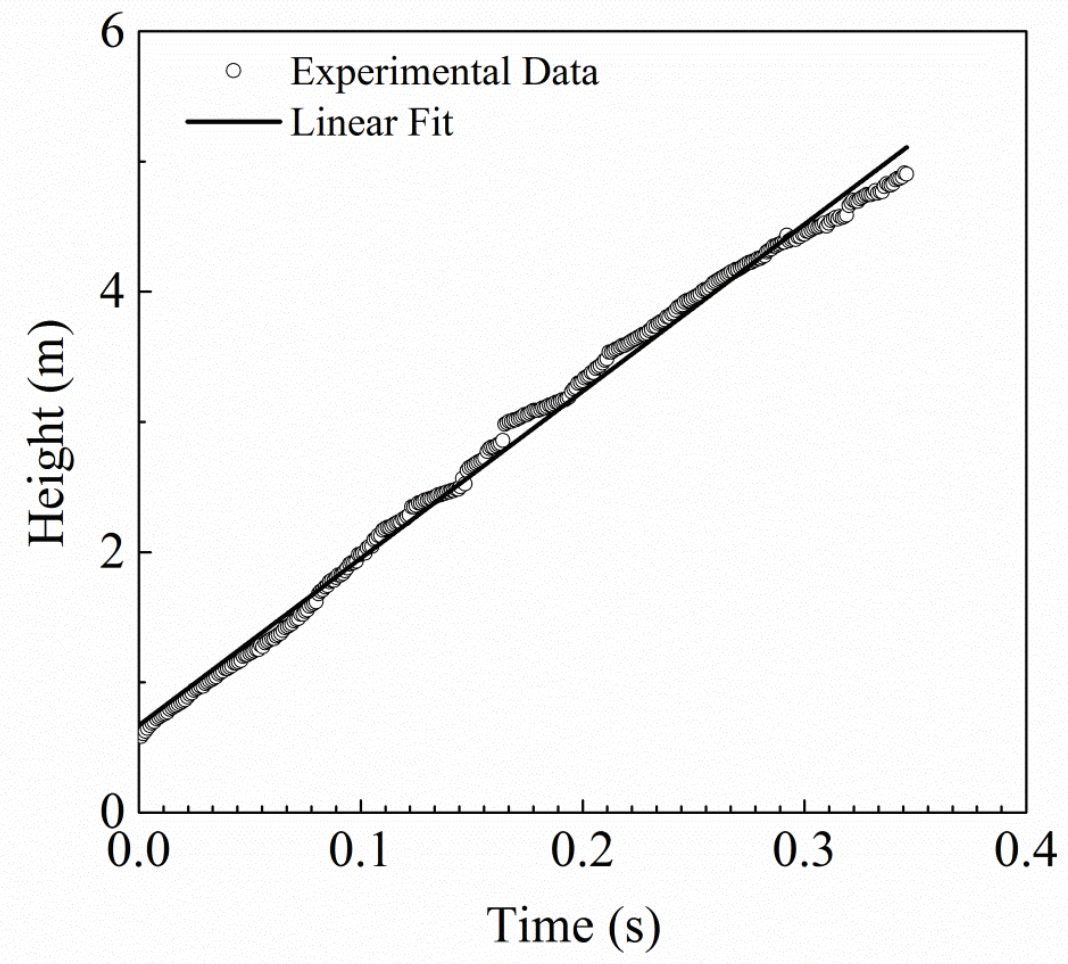

Figure 14: Flame front progression for a vertical section of the flame 


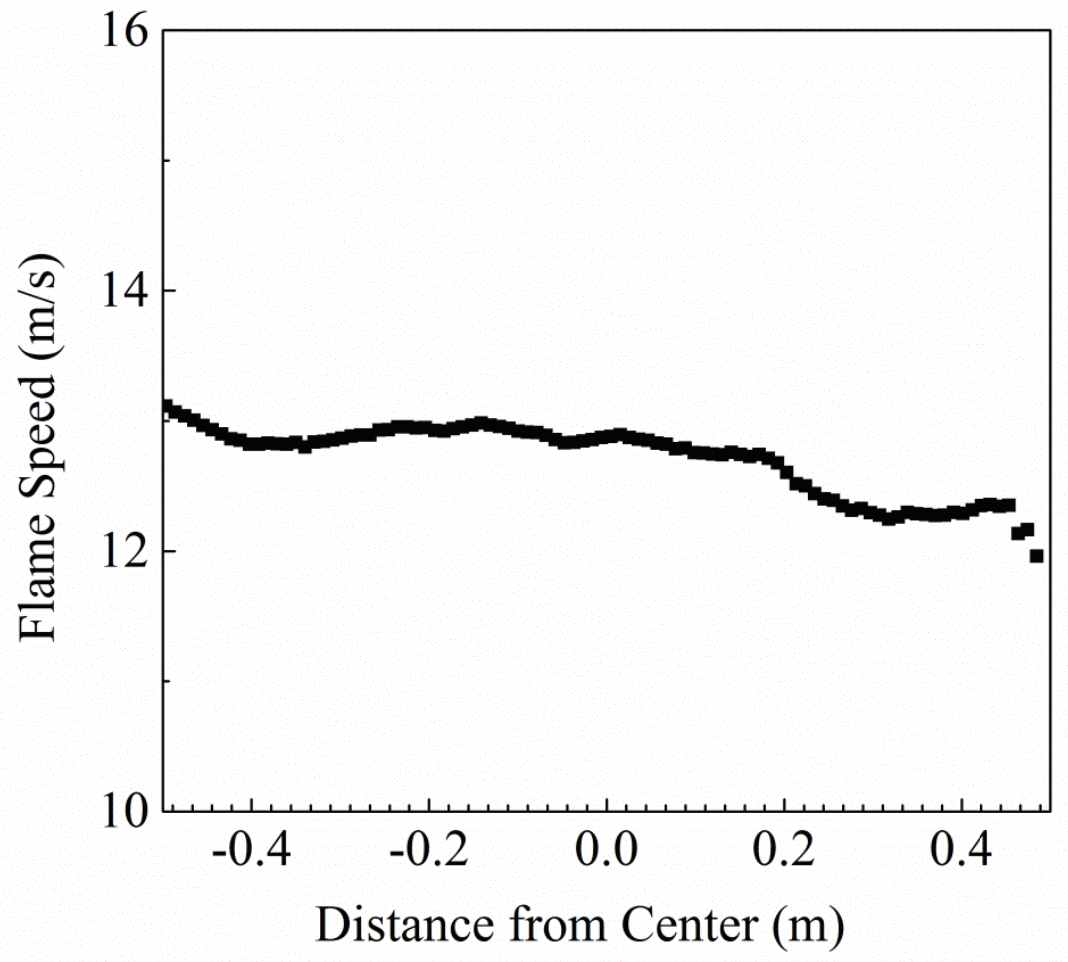

Figure 15: Calculated flame speed along the horizontal front

In total, six successful trials were performed in the field when the flame propagated without interruption from the bottom to the top of the cloud. The tests were performed with the spherical industrial aluminium powders $\mathrm{H}-5$ and $\mathrm{H}-10$ (ValimetInc, CA) having median particle diameters $d_{50}$ of $8.0 \mu \mathrm{m}$ and $12.0 \mu \mathrm{m}$, respectively. The average residual vertical velocity of the powder jet after flow cut-off and ignition can be easily deduced from the video images and is found to be around $0.2 \mathrm{~m} / \mathrm{s}$. This value has already been subtracted from the measured flame speeds shown in Table 1.

Table 1:Flame speed in large-scale aluminum dust clouds

\begin{tabular}{ccc}
\hline Trial Number & Flame Speed(m/s) & Aluminum Powder \\
\hline 1 & 13 & $\mathrm{H}-5$ \\
2 & 12 & $\mathrm{H}-5$ \\
3 & 13 & $\mathrm{H}-5$ \\
4 & 10 & $\mathrm{H}-5$ \\
5 & 14 & $\mathrm{H}-10$ \\
6 & 10 & $\mathrm{H}-10$ \\
\hline
\end{tabular}




\section{Discussion}

As mentioned above, the good agreement between the burning velocities derived from experiments with small-scale aluminium clouds having characteristic sizes from cm's (Bunsen dust flames) to tens of cm's (balloon experiments) confirms the applicability of the burning velocity notion towards small-scale dust flames. The absence of an increase in velocity with an increase in the scale of the flame over this range indicates that the effects of radiative heat transfer are negligible for small-scale dust clouds. Indeed, estimations show that the free path length of radiation in a stoichiometric aluminium dust cloud with Ampal aluminium powder is around $10 \mathrm{~cm}$ and that the absorption length is probably even larger, so that the absorption of radiation emitted by the flame and combustion products in the laboratory-scale dust clouds is effectively negligible. The radiation in such clouds acts essentially as a heat loss mechanism, similar to gas flames (Law, 2006) and, thus, can only lead to a decrease in flame speed.

However, the characteristic size of the dust cloud in the field experiments is much larger than the effective radiation absorption length, thereby ensuring that the radiation emitted by the aluminium flame front and the hot condensed combustion products will be absorbed by the fresh unburnt dust suspension. Thus, at first glance, the observed six-fold increase in flame speed in the field tests in comparison to the laboratory experiments might be considered as evidence that the flame is driven by radiative heat transfer. Simple estimations shown below, however, clearly demonstrate that the radiation alone is insufficient to explain the observed difference in flame speeds. Recent calculations (Dombrovsky, 2014) of the integral emissivity coefficient $\varepsilon$ from an infinite optically-thick layer of 0.5 micron aluminium oxide particles, which are the primary radiation emitters in aluminium flames, estimates its value at about 0.3 at $3000 \mathrm{~K}$. The flame at this temperature can then emit a maximum radiation flux $W=\varepsilon \sigma T^{4}$ of about $W=1.4 \times 10^{6}$ $\mathrm{W} / \mathrm{m}^{2}$. Because the particle residence time within the radiation heating zone $t_{r}=l / V$ (where $l$ is radiation absorption length and $V$ is the flame speed) is at least an order of magnitude longer than the characteristic time of the heat exchange between 5 micron particles and the gas, the suspension is uniformly heated by the absorbed radiation as a whole. Thus the maximum temperature increase of the aluminium suspension due to radiation absorption can be estimated from the energy balance between the enthalpy of the incoming dust flow and radiation flux, ie., $\Delta T=\frac{\varepsilon \sigma T^{4}}{\left(C_{g} \rho_{g}+C_{s} \rho_{s}\right) V}$. The estimations show that at the observed flame speeds of $10-12 \mathrm{~m} / \mathrm{s}$, the radiation preheating of the mixture would not exceed $100 \mathrm{~K}$. Such a small preheating is unable to provide a sufficient increase in the aluminium flame burning rate, irrespective of whether the particles burn in a kinetic or diffusive mode. This suggests that some other mechanism(s) besides radiative heating may be responsible for the observed high-speed flames in the field tests.

One possible mechanism is associated with the residual large-scale eddy turbulence within the dust cloud (Clavin\& Williams, 1979). The high-speed videos of the dust dispersal process in the field tests provide clear evidence of the formation of large-scale eddies generated by the highspeed submerged dust dispersal jet. Unlike the small-scale turbulence that quickly dissipates after the termination of the dispersal flow due to viscous momentum losses within two-phase media, the large eddy turbulence is more persistent. The motion of the eddies continues for several seconds after the powder is dispersed albeit with diminishing flow speeds. As was demonstrated in the theoretical work by Kagan and Sivashinsky (2000), even low-speed large- 
eddy turbulence is capable of more than an order of magnitude increase in the flame speed largely due to the increase of the flame surface area.

Another process that might lead to the increase of the flame propagation speed is specific to dust combustion and may be referred to as a "frozen turbulence" effect. Large eddies created during the dust dispersal process with a submerged jet are accompanied by the entrainment of large amounts of surrounding air into the resulting dust cloud (Yule, 1978). This leads to a stratification of the dust concentration within the jet with alternating areas of high and low dust concentration. Such stratification persists long after the decay of any turbulence due to the effectively zero diffusivity of the particulate fuel. A complex pattern of dust concentration fluctuations results in a spatially fluctuating burning interface which may lead to the appearance of a corrugated flame having an average flame speed greater than that of a freely propagating flat flame.

Of course, without direct experimental verification the proposed explanations of the effect of "live" and "frozen" large-scale eddy turbulence as a possible cause for the observed flame speed increase are unsubstantiated. One possible verification procedure would be to introduce longer delay times from the end of dust dispersal to the time of ignition of thecloud, giving the dynamic or "live" large-eddy turbulence additional time to decay. This would, however, require performing the experiments with unconfined dust clouds within a large-scale enclosure that would eliminate the influence of the weather (in particular the wind) on the stability of the dust cloud. Direct measurements of the temperature history at various locations within the cloud are also required to confirm estimations of the absence of a noticeable radiative preheating in the flame. Such experiments are already under preparation stage and will be performed in the near future.

\section{Acknowledgements}

Support for this work was provided by the Natural Sciences and Engineering Research Council of Canada and Martec, Ltd., under a Collaborative Research and Development Grant, and the Defense Threat Reduction Agency under contract HDTRA1-11-1-0014 (program manager SuhithiPeiris).

\section{References}

Cashdollar, K. L., \& Hertzberg, M. (1987).Introduction to dust explosion. In K. L. Cashdollar\& M. Hertzberg (Eds.), Industrial dust explosion (pp. 5-32): ASTM 958.

Clavin, P., Williams, F. A. (1979). Theory of premixed-flame propagation in large-scale turbulence. Journal of Fluid Mechanics, 90(03), 589-604.

Dahoe, A. E., Cant, R. S., \& Scarlett, B. (2001).On the Decay of Turbulence in the 20-Liter Explosion Sphere.Flow, Turbulence and Combustion, 67(3), 159-184.

Dombrovsky, L. A.,Emissivity of two-phase combustion products in a solid-propellant rocket engine,http://thermopedia.com/content/179/, DOI: 10.1615/thermopedia.000179, accessed September $12^{\text {th }}, 2014$. 
Goodwin, D.G. (2003). An open-source, extensible software suite for CVD process simulation. Chemical Vapor Deposition XVI and EUROCVD, 14(2003), 08.

Goroshin, S., Fomenko, I., \& Lee, J. H. S. (1996a). Burning velocities in fuel-rich aluminum dust clouds. Symposium (International) on Combustion, 26(2), 1961-1967.

Goroshin, S., Bidabadi, M., \& Lee, J. H. S. (1996b).Quenching distance of laminar flame in aluminum dust clouds.Combustion and Flame, 105(1-2), 147-160.

Goroshin, S., Mamen, J., Higgins, A.J., Bazyn, T., Glumac, N., \&Krier, H. (2007). Emission spectroscopy of flame fronts in aluminum suspensions. Proceedings of the Combustion Institute, 31(2), 2011-2019.

Goroshin, S., Tang, F.-D., Higgins, A. J., \& Lee, J. H. S. (2011). Laminar dust flames in a reduced-gravity environment. ActaAstronautica, 68(7-8), 656-666.

Gu, X. J., Haq, M. Z., Lawes, M., \& Woolley, R. (2000). Laminar burning velocity and Markstein lengths of methane-air mixtures.Combustion and Flame, 121(1-2), 41-58.

Hassan, M. I., Aung, K. T., \&Faeth, G. M. (1998). Measured and predicted properties of laminar premixed methane/air flames at various pressures. Combustion and Flame, 115(4), 539550 .

Hertzberg, M., Cashdollar, K. L., \&Zlochower, I. A. (1988). Flammability limit measurements for dusts and gases: Ignition energy requirements and pressure dependences. Proceedings of the Combustion Institute, 21(1), 303-313.

Julien, P., Soo, M., Goroshin, S., Frost, D. L., Bergthorson, J. M., Glumac, N., \& Zhang, F. (2014a). Combustion of Aluminum Suspensions in Hydrocarbon Flame Products.Journal of Propulsion and Power, 30(4), 1047-1054.

Julien, P., Whiteley, S., Goroshin, S., Soo, M., Frost, D. L., \&Bergthorson, J. M. (2014b). Flame structure and particle-combustion regimes in premixed methane-iron-air suspensions.Proceedings of the Combustion Institute, in press.

Kagan, L., \&Sivashinsky, G. (2000).Flame propagation and extinction in large-scale vortical flows.Combustion and Flame, 120(1-2), 222-232.

Kang Pu, Y., Jarosinski, J., Sheng Tai, C., William Kauffman, C., \&Sichel, M. (1989). The investigation of the feature of dispersion induced turbulence and its effects on dust explosions in closed vessels. Proceedings of the Combustion Institute, 22(1), 1777-1787.

Law, C. K. (2006). Combustion Physics.Cambrige University Press.

Palecka, J., Julien, P., Goroshin, S., Bergthorson, J. M., Frost, D. L., \& Higgins, A. J. (2014). Quenching distance of flames in hybrid methane-aluminum mixtures.Proceedings of the Combustion Institute, in press. 
Pu, Y. K., Jia, F., Wang, S. F., \&Skjold, T. (2007).Determination of the maximum effective burning velocity of dust-air mixtures in constant volume combustion.Journal of Loss Prevention in the Process Industries, 20(4-6), 462-469.

Qin, X., \&Ju, Y. (2005). Measurements of burning velocities of dimethyl ether and air premixed flames at elevated pressures. Proceedings of the Combustion Institute, 30(1), 233-240.

Skjold, T., Olsen, K. L., \& Castellanos, D. (2013). A constant pressure dust explosion experiment. Journal of Loss Prevention in the Process Industries, 26(3), 562-570.

Soo, M., Julien, P., Goroshin, S., Bergthorson, J. M., \& Frost, D. L. (2013).Stabilized flames in hybrid aluminum-methane-air mixtures.Proceedings of the Combustion Institute, 34(2), 2213-2220.

Tang, F.-D., Goroshin, S., \& Higgins, A. J. (2011). Modes of particle combustion in iron dust flames. Proceedings of the Combustion Institute, 33(2), 1975-1982.

Tang, F.-D., Goroshin, S., Higgins, A., \& Lee, J.H.S. (2009). Flame propagation and quenching in iron dust clouds. Proceedings of the Combustion Institute, 32(2), 1905-1912.

Yule, A. J. (1978). Large-scale structure in the mixing layer of a round jet.Journal of Fluid Mechanics, 89(03), 413-432. 